\title{
e-VLBI for geosciences
}

\section{Rüdiger Haas*}

Chalmers University of Technology

Department of Radio and Space Science

Onsala Space Observatory

SE-439 92 Onsala (Sweden)

E-mail: rudiger.haas@chalmers.se

This paper gives a brief overview about how e-VLBI is used today for geosciences, both operationally in offline-mode and experimentally in real-time mode. For the next generation geodetic VLBI system, called VLBI2010, e-VLBI will be necessary component and thus is part of the VLBI2010 concept. The use of e-VLBI promises the possibility to address new scientific goals in geosciences. These are related to episodic geodynamical effects that involve large mass redistribution and cause changes in Earth rotation.

Science and Technology of Long Baseline Real-Time Interferometry:

The 8th International e-VLBI Workshop, EXPReSO9

June 22 - 262009

Madrid, Spain

${ }^{*}$ Speaker. 


\section{Introduction}

Geodetic VLBI is one of the key techniques for geosciences, i.e. global geodesy and geodynamics. It is unique among the various existing geodetic space techniques since it connects directly the celestial and the terrestrial reference frames. It is thus sensitive to both frames and the transformation between the two frames. Therefore it allows to monitor positions and their temporal development in both reference frames, i.e. radio source positions in the celestial reference frame and the positions of the radio telescopes in the terrestrial reference frame, and also the complete set of transformation parameters. The latter are usually expressed and referred to as Earth rotation parameters. The Earth rotation parameters are influenced by a variety of astronomical and geophysical processes that cause Earth rotation variations over a broad spectrum of time scales. The sensitivity of geodetic VLBI to Earth rotation and reference frames makes it to be an essential technique for global geodesy and geodynamics research.

The technique is currently in a renewal phase to develop the next generation VLBI system called VLBI2010 [1]. The main goals of these efforts are an increased measurement precision and accuracy of the derived results. It is to be expected that VLBI2010 will be operational within about 2-4 years and that the VLBI technique will contribute essentially to the Global Geodetic Observing System (GGOS) of the International Association of Geodesy (IAG). The GGOS is the IAG's upcoming permanent observing system that aims at a combination and integration use of all existing and future geodetic techniques [15].

For the currently geodetic VLBI system the precision of a single group delay measurement obtained from the bandwidth synthesis method [14] can be expressed as:

$$
\sigma_{\tau}=\frac{1}{2 \cdot \pi \cdot \mathrm{SNR} \cdot \Delta \mathrm{f}_{\mathrm{rms}}}
$$

Here $\sigma_{\tau}$ is the standard deviation of the group delay $\tau$, while SNR expresses the signal-to-noise ratio of the correlation process and $\Delta \mathrm{f}_{\mathrm{rms}}$ depends on the bandwidth synthesis method.

The SNR depends on the correlation efficiency $\eta$, the efficient apertures of the two radio telescopes involved, $A_{x}$ and $A_{y}$, the bandwidth $B$, the integration time $t_{\text {int }}$, the system temperatures at the two telescopes, $T_{x}$ and $T_{y}$, the flux density of the observed radio source $F$, and Boltzmann's constant $\mathrm{k}$.

$$
\mathrm{SNR}=\eta \cdot \sqrt{\frac{\mathrm{A}_{\mathrm{x}} \cdot \mathrm{A}_{\mathrm{y}} \cdot 2 \cdot \mathrm{B} \cdot \mathrm{t}_{\text {int }}}{\mathrm{T}_{\mathrm{x}} \cdot \mathrm{T}_{\mathrm{y}}}} \cdot \frac{\mathrm{F}}{\mathrm{k}}
$$

It is clear from the above equation that the SNR can be influenced mainly by the properties of the involved observing systems, the observed bandwidth and the integration time. Atmospheric turbulence is one of the most important factors that limits the accuracy of geodetic VLBI [11]. To model atmospheric effects it is desirable to perform as many as possible observations per time unit in different directions in order to sample the turbulent atmosphere as good as possible. This means that the integration time per observation should be short and the telescopes should move fast between observations of different radio sources. The latter puts constraints on the telescope since smaller telescope can move faster that larger telescopes. For the planned VLBI2010 system telescopes of about $12 \mathrm{~m}$ diameter are discussed and one interesting idea is to have two telescopes at each site in order to allow a reliable $24 / 7$ operation, i.e. continuous observations every day [12]. 
Reducing the integration time and telescope sizes means that the bandwidth B has to be increased to reach large enough SNR. In order to achieve even higher SNR to improve the delay precision, the bandwidth has to be increased. In the VLBI2010 project data rates of $8 \mathrm{Gbps}$ in normal mode and up to $32 \mathrm{Gps}$ in burst mode are discussed [12]. Very large bandwidth might cause difficulties to store the observational data on hard drives and thus e-transfer will be necessary.

\section{Current use of e-VLBI}

The term 'e-VLBI' refers to the electronic transfer of observed VLBI data via optical fiber lines to a correlator. However, it is somewhat loosely defined and used for both a) offline e-transfer to a correlator with subsequent offline correlation and post-processing, and b) real-time e-transfer to a correlator combined with real-time correlation and near real-time data analysis.

Currently (status mid 2009), both variants are used for geodetic VLBI, however only the first one (offline e-transfer and offline correlation) is used operationally, while the second (real-time e-transfer and real-time correlation) is so far used experimentally only. In both cases the goal is to provide reliable results with low latency.

The International VLBI Service for Geodesy and Astrometry (IVS) coordinates several different observing sessions [16], including both $24 \mathrm{~h}$ sessions and short $1 \mathrm{~h}$ sessions. The $24 \mathrm{~h}$ sessions usually involve networks of about 8-12 globally distributed stations. There are different type of $24 \mathrm{~h}$ sessions focussing on different aspects. Two series, the so-called IVS-R1 and IVS-R4-series ( $\mathrm{R}$ for rapid) are observed on the first day (IVS-R1) and fourth day (IVS-R4) every week and aim at a rapid determination of the whole set of Earth rotation and orientation parameters. The goal for the R1/R4-sessions is to achieve low latency results. Other series aim e.g. at the global terrestrial reference frame (IVS-T2), regional networks (e.g. EUROPE-series, Asian-Pacific (APSG), Japanese (JADE)), the celestial reference frame (IVS-CRF), and research and development (IVS-R\&D).

The $1 \mathrm{~h}$ sessions, called intensive session (IVS-INT), aim at providing daily estimates of the Earth rotation angle (UT1-UTC). These sessions involve two stations that form a long East-West baseline. East-West oriented baselines are very sensitive for the Earth rotation angle. During weekdays (series IVS-INT-1) the involved stations are Wettzell (Germany) and Kokee (Hawaii), while during weekends (series IVS-INT-2) the involved stations are Wettzell and Tsukuba (Japan). On early Monday mornings (series IVS-INT-3) there are observations with a three station network consisting of Wettzell, Tsukuba and Ny-Ålesund (Spitzbergen).

\subsection{Offline e-transfer}

During the last 2-3 years operational e-transfer of observed VLBI data to the correlators in Bonn and Tsukuba has started. Data are recorded at the stations on standard Mark5-modules (e.g. Wettzell, Ny-Ålesund), or on a PC-EVN computer [13] that is daisy chain connected to the Mark5 data aquisition system (e.g. Onsala, [9]), or on the K5 data acquisition systems (e.g. Tsukuba). After an observing session the data are then transferred with the Tsunami transfer protocol [18] or e.g. with BBFTP [2], to a receiving computer at the correlator. Depending on the correlator and the data acquisition format at the individual stations, the data need to be converted to the format that can be handled by the correlator. Once all data of all stations participating in an observing session are available the correlation process can be started. 
Operational e-transfer is done to the Bonn correlator with the R1-data observed e.g. at Onsala, Ny-Ålesund and Tsukuba. The data from Tsukuba then need to be converted from K5 format to MarkIV format before correlation. Also the observational data of some stations participating in the EUROPE-series and T2-series (Onsala, Metsähovi) are sent to the Bonn correlator via e-transfer. For the INT-3 sessions the observed data from Wettzell, Ny-Ålesund and Tsukuba are send to the Bonn correlator, too. On the other hand, the Wettzell data of the INT2-sessions are e-transferred to the Tsukuba correlator, where they are converted to the K5-format and correlated offline.

\subsection{Real-time e-VLBI}

During 2007 a Fennoscandian-Japanese collaboration was initiated to perform real-time eVLBI observations in order to determine the Earth rotation angle (UT1-UTC) with very low latency [17]. The VLBI groups at Metsähovi (Finland), Onsala (Sweden), Kashima (Japan) and Tsukuba (Japan) organized a large number of short $1 \mathrm{~h}$ intensive-type like observing sessions where the observational data from the Fennoscandian stations are sent to correlators at Kashima and/or Tsukuba.

The Tsunami transfer protocol is used in real-time mode, and the received data are converted from Mark5 to K5-format in real-time, and fed into realtime correlation, near real-time bandwidth synthesis, and finally near real-time data analysis. The current world record for low latency was achieved in February 2008 when the final results for the Earth rotation angle were determined within 4 minutes after the end of the observing session of 1 hour duration [10].

During the summer of 2009, first successful experiments were done to do real-time e-VLBI during a complete $24 \mathrm{~h}$ VLBI-session. In session R1.385 and RD.0907, where both Onsala and Tsukuba participated, the observational data from Onsala for the baseline Onsala-Tsukuba were successfully sent in real-time to the Tsukuba correlator, converted, correlated and analyzed there during the ongoing $24 \mathrm{~h}$ observing session. The success of these efforts is very encouraging and a step towards e-VLBI with distributed correlation. Thus, there are plans to continue the efforts with further experiments during 2009.

\section{Geodynamics and Earth rotation}

The transformation between the celestial and the terrestrial reference frames is commonly referred to as Earth rotation. It is a time-varying three dimensional vector that is influenced by astronomical and geophysical processes. Usually are Earth rotation variations decomposed into three categories. These are a) variations in the actual rotation angle (or rotation speed), b) the motion of the solid Earth (i.e. the terrestrial reference frame) around the Earth rotation axis, and c) the motion of the Earth rotation axis in the inertial frame (i.e. the celestial reference frame). Variations of the rotation angle are usually expressed as variations of the difference between astronomical time UT1 and atomic time UTC, or if the rotation speed is used, this is described as variations of the so-called length-of-day (LOD). The motion of the terrestrial reference frame with respect to the Earth rotation axis is described as so-called polar motion, and the motion of the Earth rotation axis with respect to the celestial reference frame as precession and nutation.

The principle of conservation of angular momentum governs the Earth's rotational behaviour. Earth rotation variations are caused by torques acting on the solid Earth and re-distribution of 
masses that change the Earth's tensor of inertia. Relative angular momentum is for example transferred between the different sphere of the Earth, i.e. the atmosphere, hydrosphere, solid Earth and the Earth's interior, see e.g. [8], [5] [3], [7]. Mass re-distrubutions due to e.g. large earthquakes is expected to cause in Earth rotation, see e.g. [6], [4]. Earth rotation observations thus give insight to global geodynamical processes.

\section{New scientific goals: episodic geodynamical events}

New scientific goals for geodetic VLBI are episodic geodynamical events. Examples for such events are large earthquakes that change the Earth's tensor of inertia. So far the mechanisms are not fully understood and corresponding Earth rotation variations have not been observed, partly because there were no VLBI observations during such events. Other possible candidate effects to study are related to large mass re-distributions caused by volcanic eruptions and angular momentum exchange due to tropical storms.

To study such episodic effects it is necessary to be able to react flexible and fast in order to start dedicated observing sessions. Furthermore, high precision of the observations and low latency of the results is necessary and desirable. The use of e-VLBI with high precision of the VLBI observations through large observational bandwidth, the possibility to react flexible and fast on episodic events, and to achieve low latency for the results appears thus the correct approach to observe such effects.

\section{Summary and outlook}

Already today e-transfer of VLBI data is used operationally for geodetic VLBI and real-time e-VLBI is performed successfully on an experimental basis. The design of the planned VLBI2010 system includes high data rate and both offline e-transfer and real-time e-VLBI. One interesting aspect of the VLBI2010 concept is to have two telescopes at each site. This might offer the possibility to react flexibly to episodic geodynamical events, e.g earthquakes, and start dedicated observation sessions to study these phenomena. Thus, e-VLBI has the potential to help to increase knowledge in geosciences.

\section{References}

[1] Behrend D., Böhm J., Charlot P., Clark T., Corey B., Gipson J., Haas R., Koyama Y., MacMillan D., Malkin Z., Niell A., Nilsson T., Petrachenko B., Rogers A.E.E., Tuccari G., Wresnik J. (2008) "Recent Progress in the VLBI2010 Development", In: M. Sideris (ed.): Proc. of the 2007 IAG General Assembly, Perugia, Italy, July 2-13, 2007, Springer, 133(5), 833-840, ISBN/ISSN: 9783540854258.

[2] bbFTP (Big Block File Tranfer Protocol), http: / / doc . in2p3. fr/bbftp/

[3] Brzezinski A., Bizouard C., Petrov S. (2002) 'Influence of the atmosphere on earth rotation: what new can be learned from recent atmospheric angular momentum estimates?", Surveys in Geophysics, 23, 33-69.

[4] Chao B.F., Gross R.S. (2000), "Coseismic excitation of the earth's polar motion”, in: S. Dick, D. McCarthy, and B. Luzum, ASP Conference Series, 208, 335-367. 
[5] Clark T.A., Ma C., Ryan J.W., Chao B.F., Gipson J.M., MacMillan D.S., Vandenberg N.R., Eubanks T.M., Niell A.E. (1998) "Earth rotation measurement yields valuable information about the dynamics of the Earth system”, EOS, Trans. Amer. Geophys. Union, 79, 205-209.

[6] Dahlen F.A. (1973) "A correction to the excitation of the Chandler wobble by earthquakes", Geophys. J.R. Astron. Soc., 32, 203-217.

[7] de Viron O., Schwarzbaum G., Lott F., Dehant V. (2005), "Diurnal and subdiurnal effetcs of the atmosphere on the Earth rotation and geocenter motion", J. Geophys. Res., 110, B11404, doi:10.1029/2005JB003761.

[8] Dickey J.O. (1995) "Earth Rotation Variations from Hours to Centuries", In: I. Appenzeller (ed.): Highlights of Astronomy, 10, 17-44.

[9] Haas R., Wager J., Mujunen A., Ritakari J., Müskens A., Dulfer C., Bertarini A. (2007) "e-VLBI data transfer from Onsala and Metsähovi to the Bonn correlator", In: J. Böhm, A. Pany, and H. Schuh (eds.): Proc. 18th European VLBI for Geodesy and Astrometry Working Meeting, Schriftenreihe der Studienrichtung Vermessung und Geoinformation, Technische Universität Wien, 79, 27-32.

[10] Matsuzaka S., Wada K., Iwata E., Shigematsu H., Kurihara S., Morito M., Kokado K., Tanimoto D., Nozawa K. (2008) "VLBI Activities of Tsukuba 32-m Station and Tsukuba Correlator", In:

A. Finkelstein and D. Behrend (eds.): IVS 2008 General Meeting Proceedings, 98-103.

[11] Nilsson T., and Haas R. (2009) "The impact of atmospheric turbulence on geodetic VLBI", submitted to Journal of Geophysical Research.

[12] Petrachenko B., Niell A., Behrend D., Corey B., Böhm J., Charlot P., Collioud A., Gipson J., Haas R., Hobiger T., Koyama Y., MacMillan D., Malkin Z., Nilsson T., Pany A., Tuccari G., Whitney A., Wresnik J. (2008) 'Design Aspects of the VLBI2010 System - Progress Report of the IVS VLBI2010 Committee", 56 pp., available at

ftp://ivscc.gsfc.nasa.gov/pub/misc/V2C/PR-V2C_090417.pdf.

[13] Ritakari J., and Mujunen A. (2002) "A VSI-H Compatible Recording System for VLBI and e-VLBI", In: N.R. Vandenberg and K.D. Baver (eds.): IVS 2002 General Meeting Proceedings, NASA/CP-2002-210002, 128-131.

[14] Rogers A.E.E. (1970) "Very long baseline interferometry with large effective bandwidth for phase-delay measurements", Radio Science, 5(10), 1239-1247.

[15] Rummel R., Rothacher M., and Beutler G. (2005) "Integrated Global Geodetic Observing System (IGGOS) - science rationale", Journal of Geodynamics, 40, 357-362, $10.1016 / j . j \circ g .2005 .06 .003$.

[16] Schlüter W., and Behrend D. (2007) "The International VLBI Service for Geodesy and Astrometry (IVS): current capabilities and future prospects", Journal of Geodesy, 81(6-8), 379-387, DOI10.1007/s00190-006-0131-z.

[17] Sekido M., Takiguchi H., Koyama Y., Kondo T., Haas R., Wagner J., Ritakari J., Kurihara S., Kokado K. (2008) "Ultra-rapid UT1 measurements by e-VLBI", Earth Planets and Space, 60(8), 865-870.

[18] Tsunami UDP protocol, http://tsunami-udp. sourceforge.net/ 